\title{
Spin-Resonance Linewidths of Bismuth Donors in Silicon Coupled to Planar Microresonators
}

\author{
James O’Sullivan, ${ }^{1, \ddagger}$ Oscar W. Kennedy $\odot,{ }^{1, \ddagger}$ Christoph W. Zollitsch $\odot,{ }^{1}$ Mantas Šimenas, ${ }^{1}$ \\ Christopher N. Thomas $\odot,{ }^{2}$ Leonid V. Abdurakhimov $\odot,{ }^{1, \dagger}$ Stafford Withington, ${ }^{2}$ and \\ John J.L. Morton ${ }^{1,3, *}$ \\ ${ }^{1}$ London Centre for Nanotechnology, University College London, 17-19 Gordon Street, London WC1H 0AH, \\ United Kingdom \\ ${ }^{2}$ Cavendish Laboratory, University of Cambridge, JJ Thomson Avenue, Cambridge CB3 OHE, United Kingdom \\ ${ }^{3}$ Department of Electrical and Electronic Engineering, University College London, Malet Place, \\ London WC1E 7JE, United Kingdom
}

(Received 16 July 2020; revised 30 September 2020; accepted 12 November 2020; published 16 December 2020)

\begin{abstract}
Ensembles of bismuth-donor spins in silicon are promising storage elements for microwave quantum memories due to their long coherence times, which exceed seconds. The operation of an efficient quantum memory requires the achievement of critical coupling between the spin ensemble and a suitable highquality factor resonator - this in turn requires a thorough understanding of the line shapes for the relevant spin-resonance transitions, particularly considering the influence of the resonator itself on line broadening. Here, we present pulsed electron-spin-resonance measurements of ensembles of bismuth donors in natural silicon, above which niobium superconducting resonators have been patterned. By studying spin transitions across a range of frequencies and fields, we identify distinct line-broadening mechanisms and, in particular, those that can be suppressed by operating at magnetic-field-insensitive "clock transitions." Given the donor concentrations and resonator used here, we measure a cooperativity $C \sim 0.2$ and based on our findings we discuss a route to achieve unit cooperativity, as required for a quantum memory.
\end{abstract}

DOI: 10.1103/PhysRevApplied.14.064050

\section{INTRODUCTION}

Despite their impressive role in the demonstration of "quantum supremacy" [1], the coherence time of superconducting qubits remains shorter than many other solid-state systems. Complementing such superconducting quantum processors with long-lived microwave quantum memories could enhance their functionality, allowing quantum states to be stored and retrieved for further processing on much longer time scales. Ideal candidates for the storage element of quantum memories are electron spin systems, which exhibit long coherence times and possess suitable transitions even in relatively low magnetic fields, to maximize compatibility with superconducting circuits and qubits. Bismuth donors in silicon have been identified as attractive candidates against such criteria [2], as they possess a number of "clock transitions" at microwave frequencies where the coupling of donor spins to each other, and

\footnotetext{
*jjl.morton@ucl.ac.uk

$\dagger$ †resent address: NTT Basic Research Laboratories, NTT Corporation, 3-1 Morinosato-Wakamiya, Atsugi, Kanagawa 243-0198, Japan.

$¥$ These authors have contributed equally to this work.
}

to other sources of magnetic field noise, becomes heavily suppressed. At such points, coherence times of $0.1 \mathrm{~s}$ have been measured in natural silicon, rising to about $3 \mathrm{~s}$ in isotopically enriched ${ }^{28} \mathrm{Si}$ [3], over 4 orders of magnitude longer than that in state-of-the-art superconducting qubits.

Microwave quantum memories require a cavity (typically a planar superconducting resonator) either critically coupled [4] or strongly coupled [5,6] to an ensemble of paramagnetic spins, allowing for coherent exchange of quantum information. The coupling strength of the spin ensemble to the resonator, $g_{\text {ens }}$, must be sufficient to at least achieve cooperativity $C=g_{\text {ens }}^{2} / \kappa \gamma$ equal to 1 , where $\kappa$ and $\gamma$ are the half width at half maximum (HWHM) of the cavity and spin lines, respectively. In a cavity containing $N$ coherent spins with homogeneous single-spin coupling, $g_{0}$, the ensemble coupling strength, $g_{\text {ens }}$ becomes enhanced through collective coupling: $g_{\text {ens }}=g_{0} \sqrt{N}$, [7]. A variety of paramagnetic spin ensembles with spin concentrations of $10^{17-20} \mathrm{~cm}^{-3}$ have already been used to demonstrate high-cooperativity coupling to planar superconducting resonators [8-11]. For example, using phosphorus donors in isotopically purified silicon, $C \sim 2$ has been achieved; however, the coherence time, $T_{2}$, in that experiment was limited to only approximately $2 \mathrm{~ms}$, despite coherence 
times approaching seconds being measured in the same spin system in the dilute limit and in the bulk [12]. The use of clock transitions in bismuth offers the prospect of achieving sufficient coupling while maintaining long coherence times - for example, a recent study of bismuth in ${ }^{28} \mathrm{Si}$ coupled to an aluminum resonator has shown $T_{2}=$ 0.3 s combined with $C=3.5 \times 10^{-2}$ [13].

In addition to the ensemble coupling strength, which can be modified by the choice of resonator design and spin concentration, two further critical parameters are the resonator and electron-spin-resonance (ESR) linewidths. Superconducting resonators with quality factors above $10^{6}$ (corresponding to linewidths less than $10 \mathrm{kHz}$ ) have been demonstrated $[14,15]$. In contrast, ESR linewidths of bismuth donors in bulk-doped silicon have been measured at the $X$ band (approximately $9.7 \mathrm{GHz}$ ) to be $4.5 \mathrm{MHz}$ in silicon with natural isotopic abundance $\left({ }^{\text {nat }} \mathrm{Si}\right)$ and approximately $0.1 \mathrm{MHz}$ in ion-implanted isotopically enriched ${ }^{28} \mathrm{Si}[2,16]$. Thus, even for isotopically purified silicon, it is the spin linewidth that determines the threshold of the ensemble coupling strength required to enter the strongcoupling regime and, if reduced, would increase cooperativity. Furthermore, strain-induced line broadening [17] has been observed for near-surface bismuth donors underneath aluminum resonators, showing ESR line shapes dominated by inhomogeneous strain with linewidths of approximately $7 \mathrm{MHz}$ even in ${ }^{28} \mathrm{Si}$ [18].

In this paper, we study the ESR linewidths of bismuth donors in natural silicon, using niobium superconducting resonators, which are expected to induce less strain in the silicon due to a better matching of the thermal expansion coefficient. Furthermore, by examining a range of ESR transitions with different dependencies of the transition frequency $(f)$ upon the static external magnetic field strength $\left(B_{0}\right), \partial f / \partial B_{0}$ - from approximately $28 \mathrm{GHz} / \mathrm{T}$, the gyromagnetic ratio of a free electron, down the limit at which it tends to zero at a clock transition - we identify different line-broadening mechanisms and expected limits of linewidths. Based on our results, we establish how sufficiently strong coupling can be achieved between $\mathrm{Bi}$ donors and superconducting planar resonators to achieve a high-efficiency quantum memory.

\section{RESONATORS AND SPIN SYSTEM}

We fabricate resonators on a natural silicon substrate, using 100-nm-thick sputtered niobium films, deposited using parameters that minimize film-induced strain in the substrate at room temperature and patterned using a standard lift-off process. The resonator design, shown in Fig. 1(a), contains a coplanar capacitor and an inductive hairpin, where the conductor width is reduced and which is primarily responsible for coupling to spins. The hairpin geometry results in antiparallel microwave currents, confining the magnetic fields close to the resonator and improving the coupling efficiency to implanted layers of spins. The bismuth atoms are introduced into the silicon substrate, prior to resonator fabrication, by ion implantation into the top micron at a concentration of $10^{17} \mathrm{~cm}^{-3}$ [see Fig. 1(b)]. Samples are annealed at $900^{\circ} \mathrm{C}$ for $5 \mathrm{~min}$ to incorporate the bismuth atoms into the silicon lattice, forming spin-active donors with an efficiency of approximately $60 \%$ [20]. We place the resonators inside a copper $3 \mathrm{D}$ box in a setup similar to that used in Ref. [21] and measure them in transmission mode using two antennae. A full description of the fabrication and the measurement setup is given in the Supplemental Material [22].

The frequencies of the planar microresonators span a range from 6.28 to $7.55 \mathrm{GHz}$, intersecting with a number of bismuth-donor ESR transitions, as shown in Fig. 1(f). The bismuth-donor spin system comprises an electron spin $\mathbf{S}=1 / 2$ bound to the nuclear spin of ${ }^{209} \mathrm{Bi} \mathbf{I}=9 / 2$ and is described by the spin Hamiltonian in frequency units:

$$
H / h=A \mathbf{I} \cdot \mathbf{S}+\gamma_{e} \mathbf{B}_{\mathbf{0}} \cdot \mathbf{S}+\gamma_{\mathrm{Bi}} \mathbf{B}_{\mathbf{0}} \cdot \mathbf{I}
$$

where $A$ is the (isotropic) hyperfine coupling constant $(1.475 \mathrm{GHz}[23,24])$ and $\mathbf{B}_{\mathbf{0}}$ is the external magnetic field, while $\gamma_{e}=27.997$ (1) $\mathrm{GHz} / \mathrm{T}$ and $\gamma_{\mathrm{Bi}}=6.9$ (2) MHz/T are, respectively, the gyromagnetic ratios of the bound electron and the Bi nuclear spin [3]. The spin eigenstates in the low-field regime are best described by a total spin quantum number $\mathbf{F}=\mathbf{I}+\mathbf{S}$ and its projection onto $\mathbf{B}_{\mathbf{0}}$, $m_{F}$. Two classes of ESR transition arise, referred to as $S_{x}$ and $S_{z}$, which are, respectively, driven by microwave fields $\mathbf{B}_{1}$ that are perpendicular and parallel to $\mathbf{B}_{\mathbf{0}}$ [see Fig. 1(f)]. Due to the different spatial distribution of such microwave fields (simulated given approximately $50-\mathrm{nm}$ penetration depth in $\mathrm{Nb}$ [25]), as shown in Figs. 1(c) and 1(d), spins undergoing these different transitions are found in distinct physical locations around the inductor: $S_{z}$ transitions couple most strongly for spins directly beneath the resonator inductor wires and $S_{x}$ transitions for spins between the inductor wires. The different transitions obey different selection rules, where $\Delta F \Delta m_{F}= \pm 1$ for $S_{x}$ transitions and $\Delta m_{F}=0$ for $S_{z}$. The $S_{x}$ transitions come in pairs closely spaced in frequency with one transition corresponding to $\Delta F \Delta m_{F}=+1$ and another $\Delta F \Delta m_{F}=-1$. At most fields, the separation of these transitions is less than the bismuth linewidth, so they cannot be resolved. A more detailed analysis of the spin energy-level diagram is given in the Supplemental Material [22].

\section{CONTINUOUS WAVE ESR}

We first detect the ESR transitions in a continuous-wave (cw) approach by measuring microwave transmission $S_{21}$ through the microresonator, using a vector-network analyzer (VNA). Figure 2(a) shows both the quadratic decrease in the resonator frequency with an increasing 

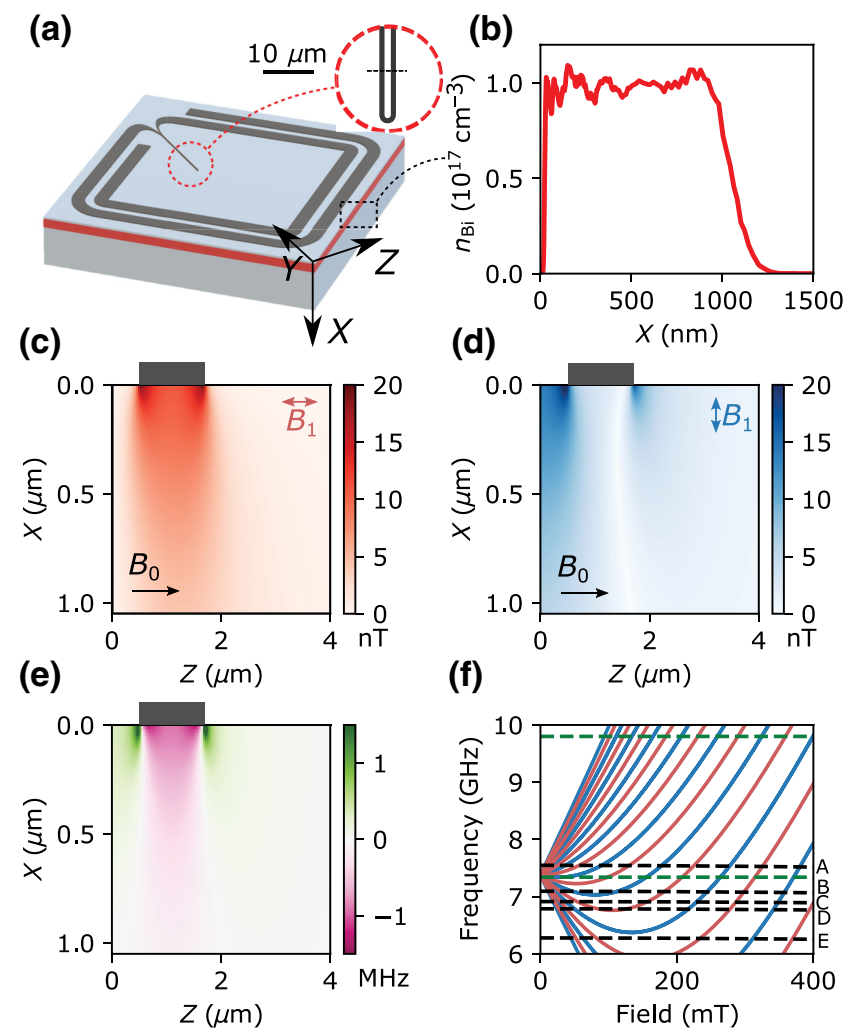

(f)

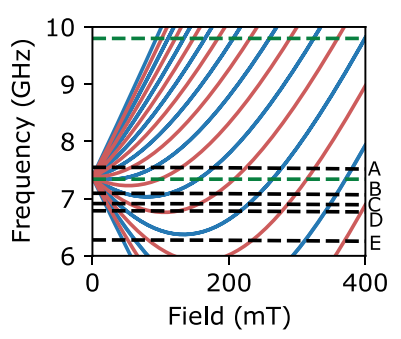

FIG. 1. (a) The layout of the resonator with a detail of the inductor, showing dashed black lines to indicate the cross sections taken in (c) and (d). The axes are shown with $X$ perpendicular to the substrate surface, $Y$ along the hairpin inductor, and $Z$ perpendicular to the inductor. (b) The implantation profile of Bi calculated by Monte Carlo simulations using the SRIM package [19]. (c),(d) Two-dimensional (2D) finite-element (FE) simulations of the microwave magnetic field $B_{1}$ due to zero-point fluctuations of the resonator current, assuming a $100 \Omega$ characteristic impedance: (c) and (d), respectively, show components that are parallel and perpendicular to the static magnetic field $B_{0}$ and, correspondingly, that drive $S_{z}$ and $S_{x}$ transitions. (e) FE simulations of shifts in the Bi donor hyperfine constant due to strain due to the mismatch of the thermal expansion coefficient between silicon and niobium. (f) The spectrum of the $S_{z}$ (red) and $S_{x}$ (blue) spin transitions in Bi-Si as a function of the magnetic field. The dashed lines indicate the frequencies of the superconducting planar (black) and nonsuperconducting three-dimensional (3D) (green) resonators used in this work. Five superconducting resonators are labeled A-E, with A (E) the highest- (lowest-) frequency resonator. There are several resonators at slightly higher frequencies than resonator $\mathrm{C}$, which are not shown here.

magnetic field (due to the increase in the kinetic inductance of the superconductor $[26,27])$, as well as a series of avoided crossings where spin transitions couple to the resonator. By fitting the microwave transmission to a Fano resonance function [28] at each magnetic field value, the shift in the resonator frequency and the reduction of the quality factor caused by coupling to the spin transition can be observed [for example, see Fig. 2(b)]. When resonant

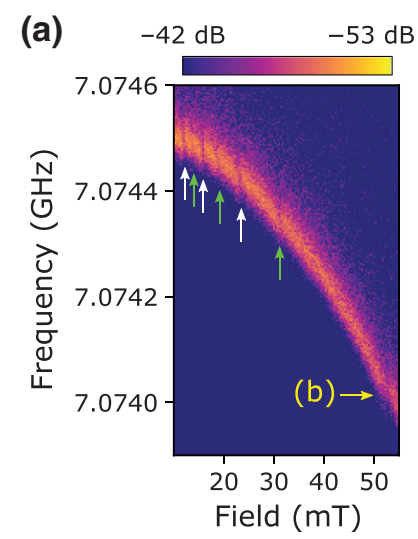

(b)

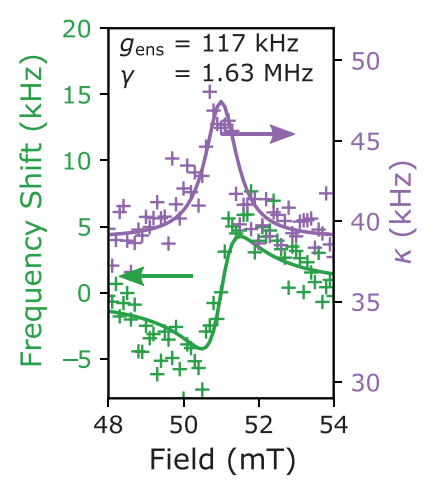

FIG. 2. (a) The field-dependent transmission $S_{21}$ through the cavity, showing the quadratic dependence of the frequency of resonator B on the magnetic field. The interactions between spins and the resonator are highlighted with arrows, where white (green) arrows identify $S_{x}\left(S_{z}\right)$ transitions. The $S_{x}$ transition shown in (b) is labeled with a yellow arrow. (b) The fit to cw data of an $S_{x}$ transition with $\partial f / \partial B_{0}=-0.11 \gamma_{e}$, showing an ensemble coupling strength of $120 \mathrm{kHz}$ and a cooperativity of 0.2 . The temperature is $140 \mathrm{mK}$.

with a spin transition the measured resonator linewidth $\kappa$, and frequency, $f_{\text {res }}$, are determined by the spin line width and ensemble coupling [29]:

$$
\begin{gathered}
\kappa=\kappa_{0}+\frac{g_{\text {ens }}^{2} \gamma}{\Delta^{2}+\gamma^{2}}, \\
f=f_{0}-\frac{g_{\text {ens }}^{2} \Delta}{\Delta^{2}+\gamma^{2}},
\end{gathered}
$$

where $\kappa_{0}$ and $f_{0}$ are, respectively, the half width and frequency of the resonator in the absence of spins and the detuning $\Delta=\left(B_{0}-B_{R}\right) \times \partial f / \partial B_{0}$, where $B_{R}$ is the magnetic field at which the spins and the resonator are resonant. The cw data therefore give a measure of the spin linewidth and also a measure of ensemble coupling strength - for example, fits to the data shown in Fig. 2(b) give $g_{\text {ens }}=$ $120(10) \mathrm{kHz}$ and $\gamma=1.6(2) \mathrm{MHz}$. Together, these result in a cooperativity $C \sim 0.2$ that, while the highest value reported thus far for bismuth donors, is a factor of 5 too small to achieve critical coupling.

\section{PULSED ESR}

To measure the spin-resonance line shape in greater detail, the magnetic field is swept as pulsed ESR measurements are performed at the resonator frequency. A CarrPurcell-Meiboom-Gill (CPMG) pulse sequence [30,31] is applied for the purposes of "echo-train averaging" [32] and the emitted electron spin-echo signal is amplified at multiple stages and captured on a digitizer (full details of the 
spectrometer and averaging scheme are shown in the Supplemental Material [22]). The echo amplitude measured as a function of the magnetic field in an echo-detected field sweep (EDFS) is shown for a representative $S_{z}$ transition $\left(6.8 \mathrm{GHz}, \partial f / \partial B_{0}=-0.12 \gamma_{e}\right)$ in Fig. 3(a). These line shapes are well described by a sum of Gaussians with centers determined by shifts to the hyperfine constant due to the average mass of nearest-neighbor silicon atoms weighted by the probability of each configuration of neighbors based on the natural isotopic abundance [33]. Given that the relative amplitude and field shift for each of these additional peaks is fixed and known, we only need two free parameters for the fit: the central magnetic field and the common width of the constituent Gaussians (for more details, see the Supplemental Material [22]). The line widths (half widths at half maximum) extracted in this way are shown in Fig. 3(c) for a number of $S_{x}$ and $S_{z}$ transitions, plotted as a function of the first-order magnetic field sensitivity, $\partial f / \partial B_{0}$, for each transition. The linear dependence of the linewidth upon $\partial f / \partial B_{0}$ implies approximately $0.5 \mathrm{mT}$ variations in effective magnetic field, arising from random distributions in ${ }^{29} \mathrm{Si}$ nuclei.

Of particular interest is the limiting case in which $\partial f / \partial B_{0} \sim 0$, for example around the $6.76 \mathrm{GHz} S_{z}$ "clock transition," where Fig. 3(b) shows the echo-detected magnetic field sweep as resonator $\mathrm{C}$ is tuned downward in frequency by tilting the applied field with respect to the resonator plane [14]. EDFSs are collected at each $B_{0}$ as the resonator is tuned and are also shown in Fig. 3(b). When the resonator is at a higher frequency (with the field perfectly aligned in the resonator plane), distinct peaks in echo amplitude on either side of the clock transition are clearly resolved. As the resonator frequency is tuned downward, toward the clock transition, these peaks merge. As in Ref. [3], we can use the clock transition to precisely determine the value of the hyperfine constant in this sample and we find that $A=1475.31 \mathrm{MHz}, 140 \mathrm{kHz}$ higher than measured in ${ }^{28} \mathrm{Si}$, attributed to the larger average mass of silicon atoms, which is expected to increase the hyperfine constant by approximately $160 \mathrm{kHz}$ [33]. In none of these spectra are individual nearest-neighbor mass-shifted peaks resolvable, implying that broadening of at least $0.85 \mathrm{MHz}$ remains in these devices. A minimum linewidth of approximately $1.25 \mathrm{MHz}$ is inferred from the fits, which, based on the value of $\partial f / \partial A$ for this transition, equates to a HWHM in $A$ of $250 \mathrm{kHz}$, substantially greater than the value of $30 \mathrm{kHz}$ measured in low-density bulk-doped $\mathrm{Bi}-{ }^{\text {nat }} \mathrm{Si}$ at a clock transition [3].

In order to distinguish between potential contributions to the broadening seen here (such as ion-implantation damage or strain from the microresonator), we measure linewidths in the ion-implanted sample using 3D microwave resonators (a sapphire ring at $9.66 \mathrm{GHz}$ and a copper loop-gap resonator at $7.3386 \mathrm{GHz}$ ). Far from the clock transition, the measurements from the $3 \mathrm{D}$ resonator match well with (a)

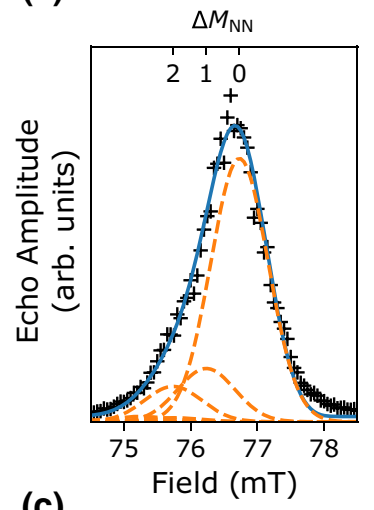

(b)

(c)
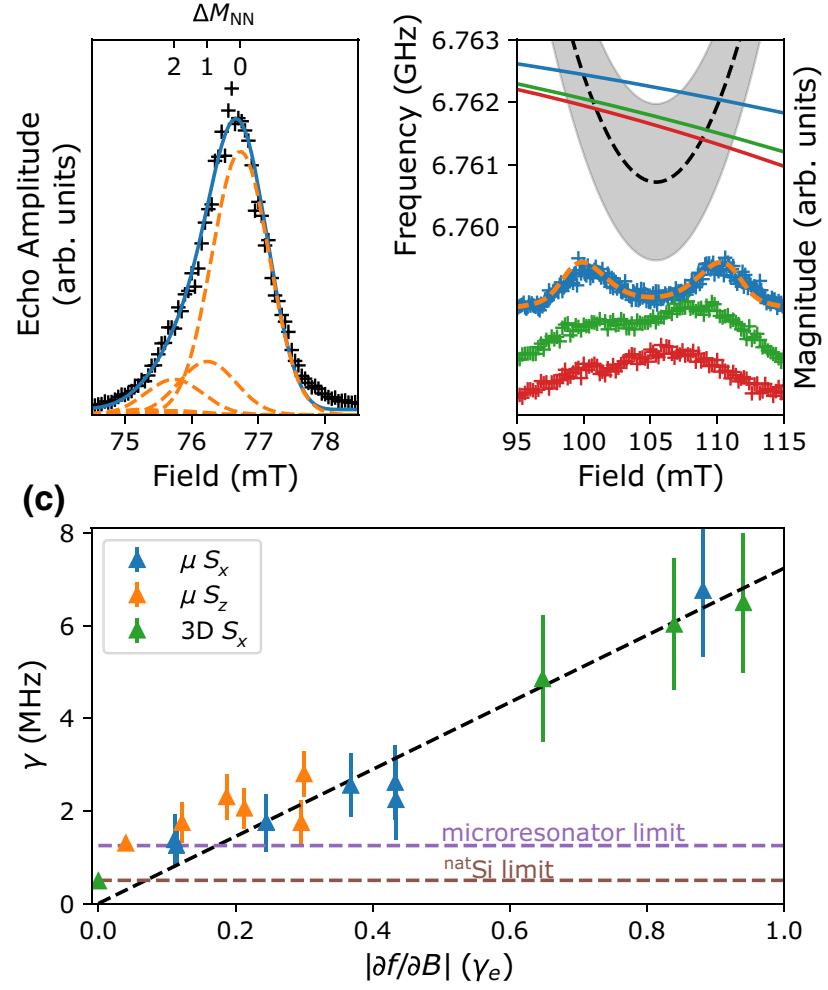

FIG. 3. (a) The EDFS of a resonator with a slightly higher frequency than that of resonator $\mathrm{C}$ crossing an $S_{z}$ transition with $\partial f / \partial B_{0}=-0.12 \gamma_{e}$ and a fit considering known effects from nearest-neighbor silicon isotopes. (b) The EDFS at an $S_{z}$ clock transition, where resonator $\mathrm{C}$ has been tuned downward, showing well-resolved split peaks that merge when the resonator is approaching clock transition. The solid lines show fits to the (weakly field-dependent) resonator frequency and the dashed black line shows the spin transition with the $1.25-\mathrm{MHz}$ full width at half maximum of the inhomogeneously broadened line shaded in gray about the spin line. The dashed orange line overlaid on the data shows a fit to the EDFS considering nearest-neighbor masses, giving an intrinsic linewidth of $1.25 \mathrm{MHz}$. (c) Aggregate data (all resonators) showing the widths of individual Gaussians from fits as in (a) and (b) to transitions measured with different $\partial f / \partial B_{0}$, including $S_{x}$ and $S_{z}$ transitions measured using planar microresonators, as well as $S_{x}$ transitions measured in a 3D cavity at the $X$ band $(9.66 \mathrm{GHz}$ and $12 \mathrm{~K})$ and a clock transition (7.3386 GHz and $10 \mathrm{~K})$. CPMG-based averaging of 100 echoes is used to enhance the signal-to-noise ratio.

the results from the microresonators, while at the 7.3 $\mathrm{GHz}$ clock transition a linewidth of approximately 0.5 $\mathrm{MHz}$ is seen, equivalent to a HWHM in $A$ of $0.1 \mathrm{MHz}$, enabling $\mathrm{Si}$ isotope shifts to be resolved (see the Supplemental Material [22]). The difference in the distribution of hyperfine couplings from bulk-doped $\mathrm{Si}$ and the ionimplanted samples studied here could be caused either by the $100 \times$ higher bismuth-donor concentration and/or residual strain from ion implantation [20]. For spins in silicon beneath patterned microresonators, strain is known 
to arise from the different coefficients of thermal expansion (CTEs) of the various materials. While previous observations of microresonator-induced strain have been based on aluminum [18,21], the niobium material used here has a CTE that is better matched to that of silicon. As a result, we observe shifts in the hyperfine constant that are approximately half of those seen for aluminum resonators [18], despite these aluminum resonators having a minimum feature size that is four times larger than the resonators in this work.

Due to the inhomogeneous coupling between spins and the microresonator, different spatial distributions of $\mathrm{Bi}$ spins can be addressed by varying the power used in pulsed ESR measurements [34]. For example, measurements taken with lower microwave powers should be more sensitive to spins that are closer (and thus, more strongly coupled) to the resonator. In order to investigate the spatial distribution of the ESR line broadening, we perform power-dependent pulsed ESR measurements and compare the results with 2D FE simulations of the strain distribution and expected ESR signals [see Fig. 4]. Despite this $B_{1}$ inhomogeneity, uniform global manipulation of the spin ensemble remains possible using techniques such as adiabatic fast-passage $[35,36]$.

We apply a $2-\mu$ s pulse of variable amplitude, followed some time $t=10 \mathrm{~ms}$ later (where $T_{2} \ll t \ll T_{1}$ ) by a two-pulse Hahn echo-detection sequence with pulses at $2 \mu \mathrm{s}$ and $4 \mu \mathrm{s}$, respectively, and $\tau=60 \mu \mathrm{s}$, as shown in Fig. 4(e). In these samples, $T_{2}$ varies depending on the transition and shot repetition time, with all coherence lost after waits longer than $1 \mathrm{~ms}$, a detailed study of which is the subject of future work. $T_{1}$ varies between donors and is determined by the Purcell effect $[37,38]$, with virtually all spins having $T_{1}>100 \mathrm{~ms}$. Using a low "detection" power for the Hahn echo sequence $(-26 \mathrm{dBm}$ at the source, which is amplified and attenuated, giving approximately -26 $\mathrm{dBm}$ at the input antenna and further attenuated by insertion loss; for details, see the Supplemental Material [22]), we observe damped Rabi oscillations as expected, given the large range of microwave magnetic field strengths $\left(B_{1}\right)$ experienced by different spins. The detection power determines the distribution of spins contributing to the observed signal, as illustrated in the 2D simulations shown in [Fig. 4(a)]. The contribution of each pixel goes as $g_{0} \sin ^{3}[\theta(\mathbf{r})]$ [39], where $\theta(\mathbf{r})=\gamma_{e} B_{1}(\mathbf{r}) A_{\text {pulse }} t_{\text {pulse }}$ is the spatially varying tip angle induced by the first pulse in the Hahn echo sequence of amplitude $A_{\text {pulse }}$ and duration $t_{\text {pulse }}$.

Having selected a subensemble of spins through the choice of "detection" power in the Hahn echo, we can then understand the effect of sweeping the amplitude of the initial pulse. The simulated projection $S_{z}$ of different spins following this initial pulse is shown in Figs. 4(b)-4(d) for different pulse amplitudes: at lower amplitudes, only those spins closest to the resonator are flipped, while for larger amplitudes multiple bands of spins can be identified,
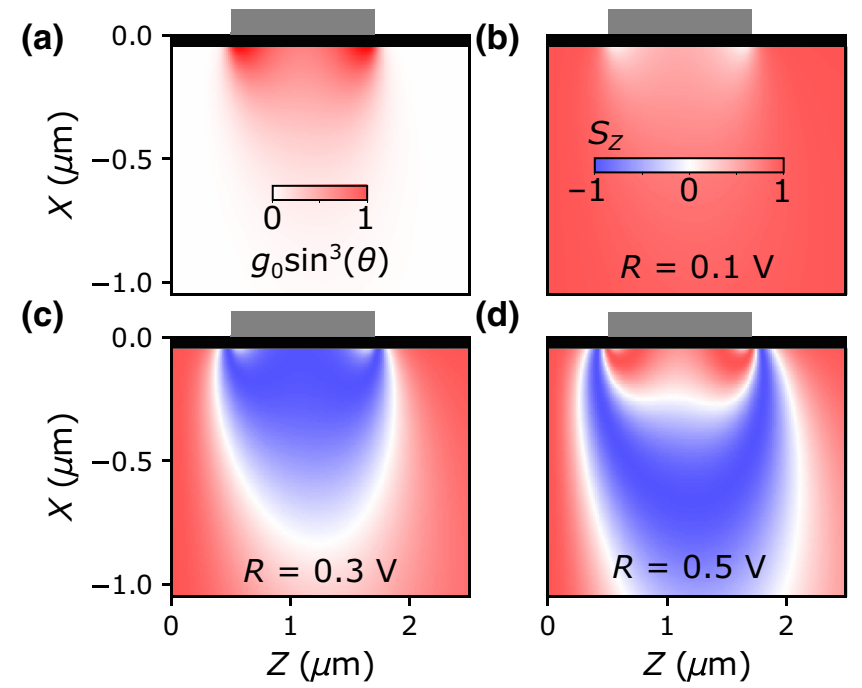

(d)

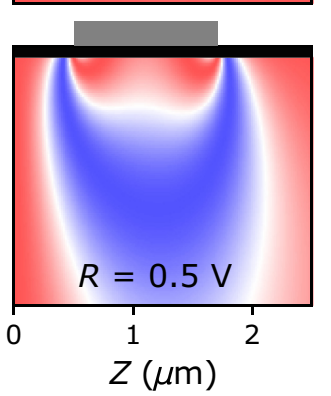

(e)

(f)
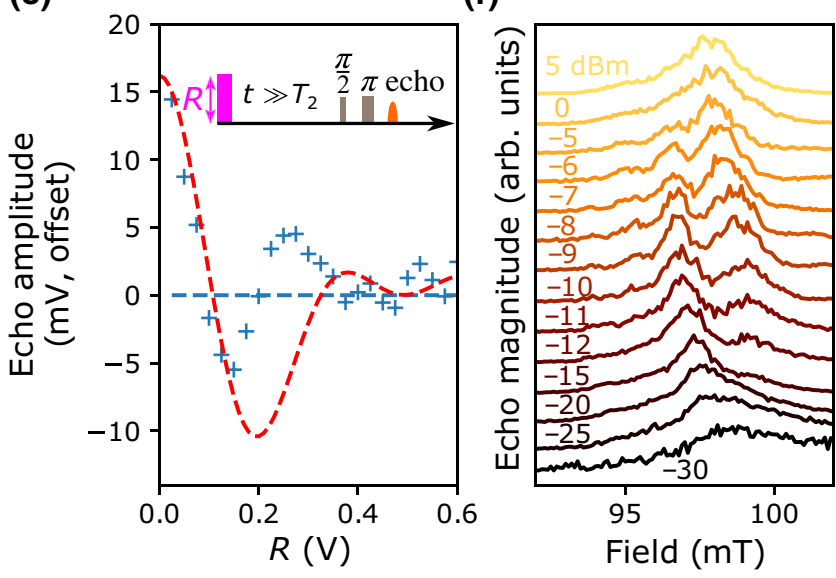

FIG. 4. Simulations of the echo amplitude are performed by multiplying a contribution of each pixel (a) by the projection of the pixel along $z$ (b)-(d) and by integrating over pixels. In (a)-(d), the resonator cross section is indicated in gray and donors assumed to be ionized due to band bending are shown in black. (a) The calculated contribution of each pixel to a spin echo measured using a two-pulse Hahn echo sequence $(\pi / 2-$ $t-\pi-t-$ echo). The pulse amplitude is chosen to best match the data obtained, using a power of about $-26 \mathrm{dBm}$ at the input antenna (estimated at approximately $5 \times 10^{5}$ photons in the resonator-see Sec. G in the Supplemental Material [22]). (b)-(d) Simulated spatial variations of the $z$ projection of spins following a rectangular pulse of amplitude $R=0.1 \mathrm{~V}, R=0.3 \mathrm{~V}$, and $R=0.5 \mathrm{~V}$. (e) Experimental (blue crosses) and simulated (red dashed line) Rabi oscillations collected on resonator $\mathrm{C}$ at the $S_{z}$ clock transition shown in Fig. 3(b) (microwave power -26 $\mathrm{dBm})$. The inset pulse sequence shows the first rotation pulse in magenta, the amplitude of which is swept in the Rabi experiment. (f) Power-dependent echo-detected field sweeps collected with a CPMG-1000 sequence on resonator C.

corresponding to spins having been rotated by even or odd multiples of $\pi$. Summing over all cells, weighted by their contribution to the detection Hahn-echo signal 
[Fig. 4(a)], gives the simulated Rabi oscillations shown in Fig. 4(e). The qualitative agreement between the simulated and experimental pulsed ESR measurements at low detection powers suggests that the simulations can be used to understand the microscopic distribution of spins contributing to the signal in a given ESR experiment, with spins closest to the resonator contributing most to experiments performed with lower power.

By varying the detection power, we can obtain spin line widths and line shapes for different spatial distributions of spins [40], as shown in Fig. 4(f). At the highest drive power of $+5 \mathrm{dBm}$ at the source, giving approximately $5 \mathrm{dBm}$ at the input antenna (as used in Fig. 3), the echo contains contributions from many interfering bands of spins across a wide cross-section area of silicon beneath the resonator, extending across the implanted spins (see Figs. S7 and S8 in the Supplemental Material [22]). Reduction of the detection power reduces the distribution of spins and leads to narrower features in the echo-detected field-swept spectra. Oscillations in such spectra arise as different bands of spatially distributed spins, with distinct strain-shifted hyperfine constants, are tuned onto resonance at different magnetic fields. The half width of these features is approximately $0.5 \mathrm{MHz}$, similar to that seen in measurements using 3D cavities, confirming that narrower linewidths can be obtained by sampling a suitable subset of the spins to minimize the effect of strain broadening and that magnetic field broadening is not increased in these devices relative to the 3D-cavity measurement. At the lowest powers (probing spins closest to the resonator), the spectrum becomes a single broad peak - although the spatial distribution is now very narrow, it corresponds to the region of highest strain, that immediately next to the resonator. Further details and simulations of these power-dependent magnetic field spectra are presented in the Supplemental Material [22], along with arguments ruling out the Meissner effect as a contribution to line broadening for near-surface donors.

\section{OUTLOOK AND CONCLUSIONS}

A variety of approaches exist for increasing the cooperativity from the value of 0.2 measured here to the levels required to achieve a bismuth-donor spin microwave quantum memory. The first is simply to increase the density of the implanted bismuth. While activation of the Bi donor spins can become challenging at donor densities above $3 \times 10^{17} \mathrm{~cm}^{-3}$, such a concentration would already provide a sufficient increase in the spin number to reach a unit cooperativity that enables quantum memory through a "critical coupling" protocol [4]. Additionally, the proportion of resonant bismuth donors can be increased by adjusting the nuclear spin populations from their thermal equilibrium values using, for example, resonant radiofrequency excitation [41] or optical pumping [41,42]. Such an approach could increase the number of resonant spins by over 12 times the equilibrium value.

As well as increasing the ensemble coupling strength through the number of resonant spins, we consider techniques to reduce the linewidth of the bismuth-donor spins. The use of ${ }^{\mathrm{Nat}} \mathrm{Si}$ means that there are multiple nearestneighbor mass shifts to the Bi hyperfine constant and the only $72 \%$ of the Bi has all four neighbors as ${ }^{28} \mathrm{Si}$. The use of isotopically purified $\mathrm{Si}$ would concentrate all the Bi donors into a single nearest-neighbor configuration and increase the resonant spin density by approximately $40 \%$. Operating at a clock transition enables a narrowing of the linewidth of each nearest-neighbor peak down to a value of $1.25 \mathrm{MHz}$ limited by strain, independent of isotopic purification. A reduction of the strain in the silicon directly beneath the patterned microresonators could be achieved either by control of the sputtering parameters $[43,44]$ or by cryogenically cooling substrates during deposition. The elimination of strain in these devices could reduce the minimum spin linewidth by a factor of 2.5 (the difference in linewidth between the patterned and unpatterned samples) and an understanding of the broadening mechanisms behind the linewidth of approximately $0.5 \mathrm{MHz}$ measured in a $3 \mathrm{D}$ cavity could lead to further improvements. The use of isotopically purified silicon would remove nearestneighbor mass shifts, which also broaden the line. Each of the above strategies is likely to have an impact on the coherence time of the memory due to the impact in terms of concentration of resonator donor spins.

The cooperativity is also determined by the loss rate from the resonator, given by the sum of $\kappa_{i}$, the internal loss rate of the resonator, and $\kappa_{c}$, the coupling loss rate of the resonator. For spin-echo experiments that form the basis of quantum memory protocols, we require $\kappa_{c} \gg \kappa_{i}$ such that when a spin-echo forms, it can be coupled to a microwave bus rather than lost to the resonator environment. Stateof-the-art planar microwave resonators have single-photon quality factors in excess of 1 million [15], suggesting a target coupled resonator quality factor of 10000 in order to achieve a memory efficiency of 0.99 . In this work, the internal $Q$ factor is approximately 100000 , with a coupled $Q$ factor of approximately 30000 , meaning that this device is suboptimally coupled for a high efficiency quantum memory. In future devices, higher internal quality-factor resonators - such as those already demonstrated at fields compatible with bismuth clock transitions [14,45] — could give high-efficiency (0.97) memories without impacting the cooperativity measured here. The cooperativity in the narrow- $\kappa$ limit is discussed in the Supplemental Material [22] and in Refs. [46,47].

In conclusion, we measure the linewidth of spin transitions in $\mathrm{Bi}-\mathrm{Si}$ in devices compatible with quantum memory protocols. We find that upon approaching clock transitions, the linewidth of bismuth-donor spin transitions narrows to $1.25 \mathrm{MHz}$ for superconducting planar 
microresonators patterned upon the doped silicon and 0.5 $\mathrm{MHz}$ in unpatterned devices. We show that the linewidth limit in microresonators is caused by strain at the metalsilicon interface and, based on our results, we map out a route to achieving sufficiently strong coupling to develop microwave quantum memories based on bismuth-donor spins in silicon.

\section{ACKNOWLEDGMENTS}

We acknowledge the United Kingdom (U.K.) National Ion Beam Centre (UKNIBC), where the silicon samples were ion implanted, and Nianhua Peng, who performed the ion implantation. This project has received funding from the U.K. Engineering and Physical Sciences Research Council, through University College London Quantum (UCLQ) postdoctoral fellowships (O.W.K. and M.S.; Grant No. EP/P510270/1) and a Doctoral Training Grant (J.O'S.). J.J.L.M. acknowledges funding from the European Research Council under the European Union's Horizon 2020 research and innovation program [Grant Agreement No. 771493 (LOQO-MOTIONS)].

[1] F. Arute, K. Arya, R. Babbush, D. Bacon, J. C. Bardin, R. Barends, R. Biswas, S. Boixo, F. G. Brandao, and D. A. Buell et al., Quantum supremacy using a programmable superconducting processor, Nature 574, 505 (2019).

[2] R. E. George, W. Witzel, H. Riemann, N. Abrosimov, N. Nötzel, M. L. Thewalt, and J. J. Morton, Electron Spin Coherence and Electron Nuclear Double Resonance of Bi Donors in Natural Si, Phys. Rev. Lett. 105, 067601 (2010).

[3] G. Wolfowicz, A. M. Tyryshkin, R. E. George, H. Riemann, N. V. Abrosimov, P. Becker, H.-J. Pohl, M. L. Thewalt, S. A. Lyon, and J. J. Morton, Atomic clock transitions in silicon-based spin qubits, Nat. Nanotechnol. 8, 561 (2013).

[4] M. Afzelius, N. Sangouard, G. Johansson, M. Staudt, and C. Wilson, Proposal for a coherent quantum memory for propagating microwave photons, New J. Phys. 15, 065008 (2013).

[5] B. Julsgaard, C. Grezes, P. Bertet, and K. Mølmer, Quantum Memory for Microwave Photons in an Inhomogeneously Broadened Spin Ensemble, Phys. Rev. Lett. 110, 250503 (2013).

[6] C. Grezes, Y. Kubo, B. Julsgaard, T. Umeda, J. Isoya, H. Sumiya, H. Abe, S. Onoda, T. Ohshima, K. Nakamura, I. Diniz, A. Auffeves, V. Jacques, J.-F. Roch, D. Vion, D. Esteve, K. Moelmer, and P. Bertet, Towards a spinensemble quantum memory for superconducting qubits, $\mathrm{C}$. R. Phys. 17, 693 (2016).

[7] M. Tavis and F. W. Cummings, Approximate solutions for an $N$-molecule-radiation-field Hamiltonian, Phys. Rev. 188, 692 (1969).

[8] Y. Kubo, F. Ong, P. Bertet, D. Vion, V. Jacques, D. Zheng, A. Dréau, J.-F. Roch, A. Auffèves, F. Jelezko, J. Wrachtrup, M. Barthe, P. Bergonzo, and D. Esteve, Strong Coupling of a Spin Ensemble to a Superconducting Resonator, Phys. Rev. Lett. 105, 140502 (2010).

[9] S. Weichselbaumer, M. Zens, C. W. Zollitsch, M. S. Brandt, S. Rotter, R. Gross, and H. Huebl, Echo Trains in Pulsed Electron Spin Resonance of a Strongly Coupled Spin Ensemble, Phys. Rev. Lett. 125, 137701 (2020).

[10] S. Probst, H. Rotzinger, S. Wünsch, P. Jung, M. Jerger, M. Siegel, A. Ustinov, and P. Bushev, Anisotropic Rare-Earth Spin Ensemble Strongly Coupled to a Superconducting Resonator, Phys. Rev. Lett. 110, 157001 (2013).

[11] D. Schuster, A. Sears, E. Ginossar, L. DiCarlo, L. Frunzio, J. Morton, H. Wu, G. Briggs, B. Buckley, D. Awschalom, and R. Schoelkopf, High-Cooperativity Coupling of Electron-Spin Ensembles to Superconducting Cavities, Phys. Rev. Lett. 105, 140501 (2010).

[12] P. Ross, B. C. Rose, C. C. Lo, M. L. Thewalt, A. M. Tyryshkin, S. A. Lyon, and J. J. Morton, Electron Spin Resonance of P Donors in Isotopically Purified Si Detected by Contactless Photoconductivity, Phys. Rev. Appl. 11, 054014 (2019).

[13] V. Ranjan, J. O'Sullivan, E. Albertinale, B. Albanese, T. Chanelière, T. Schenkel, D. Vion, D. Esteve, E. Flurin, J. J. L. Morton, and P. Bertet, Multimode storage of quantum microwave fields in electron spins over $100 \mathrm{~ms}$, arXiv:2005.09275 [quant-ph] (2020).

[14] C. W. Zollitsch, J. O'Sullivan, O. Kennedy, G. Dold, and J. J. Morton, Tuning high- $Q$ superconducting resonators by magnetic field reorientation, AIP Adv. 9, 125225 (2019).

[15] A. Megrant, C. Neill, R. Barends, B. Chiaro, Y. Chen, L. Feigl, J. Kelly, E. Lucero, M. Mariantoni, P. J. J. O’Malley, D. Sank, A. Vainsencher, J. Wenner, T. C. White, Y. Yin, J. Zhao, C. J. Palmstrøm, J. M. Martinis, and A. N. Cleland, Planar superconducting resonators with internal quality factors above one million, Appl. Phys. Lett. 100, 113510 (2012)

[16] C. Weis, C. Lo, V. Lang, A. Tyryshkin, R. George, K. Yu, J. Bokor, S. A. Lyon, J. Morton, and T. Schenkel, Electrical activation and electron spin resonance measurements of implanted bismuth in isotopically enriched silicon-28, Appl. Phys. Lett. 100, 172104 (2012).

[17] J. Mansir, P. Conti, Z. Zeng, J. J. Pla, P. Bertet, M. W. Swift, C. G. Van de Walle, M. L. Thewalt, B. Sklenard, Y.-M. Niquet, and J. J. Morton, Linear Hyperfine Tuning of Donor Spins in Silicon Using Hydrostatic Strain, Phys. Rev. Lett. 120, 167701 (2018).

[18] J. Pla, A. Bienfait, G. Pica, J. Mansir, F. Mohiyaddin, Z. Zeng, Y.-M. Niquet, A. Morello, T. Schenkel, J. Morton, and P. Bertet, Strain-Induced Spin-Resonance Shifts in Silicon Devices, Phys. Rev. Appl. 9, 044014 (2018).

[19] J. F. Ziegler, M. D. Ziegler, and J. P. Biersack, SRIM-The stopping and range of ions in matter (2010), Nucl. Instrum. Methods Phys. Res. Sec. B: Beam Interact. Mater. At. 268, 1818 (2010).

[20] T. Peach, K. Homewood, M. Lourenco, M. Hughes, K. Saeedi, N. Stavrias, J. Li, S. Chick, B. Murdin, and S. Clowes, The effect of lattice damage and annealing conditions on the hyperfine structure of ion implanted bismuth donors in silicon, Adv. Quantum Technol. 1, 1800038 (2018). 
[21] A. Bienfait, J. Pla, Y. Kubo, M. Stern, X. Zhou, C. Lo, C. Weis, T. Schenkel, M. Thewalt, and D. Vion et al., Reaching the quantum limit of sensitivity in electron spin resonance, Nat. Nanotechnol. 11, 253 (2016).

[22] See the Supplemental Material at http://link.aps.org/supple mental/10.1103/PhysRevApplied.14.064050 for additional experimental details.

[23] G. Feher, Electron spin resonance experiments on donors in silicon. I. Electronic structure of donors by the electron nuclear double resonance technique, Phys. Rev. 114, 1219 (1959).

[24] G. W. Morley, M. Warner, A. M. Stoneham, P. T. Greenland, J. Van Tol, C. W. Kay, and G. Aeppli, The initialization and manipulation of quantum information stored in silicon by bismuth dopants, Nat. Mater. 9, 725 (2010).

[25] B. W. Maxfield and W. McLean, Superconducting penetration depth of niobium, Phys. Rev. 139, A1515 (1965).

[26] N. Groll, A. Gurevich, and I. Chiorescu, Measurement of the nonlinear Meissner effect in superconducting $\mathrm{Nb}$ films using a resonant microwave cavity: A probe of unconventional pairing symmetries, Phys. Rev. B 81, 020504 (2010).

[27] S. Yip and J. Sauls, Nonlinear Meissner Effect in $\mathrm{CuO}$ Superconductors, Phys. Rev. Lett. 69, 2264 (1992).

[28] U. Fano, Effects of configuration interaction on intensities and phase shifts, Phys. Rev. 124, 1866 (1961).

[29] E. Abe, H. Wu, A. Ardavan, and J. J. L. Morton, Electron spin ensemble strongly coupled to a three-dimensional microwave cavity, Appl. Phys. Lett. 98, 251108 (2011).

[30] H. Y. Carr and E. M. Purcell, Effects of diffusion on free precession in nuclear magnetic resonance experiments, Phys. Rev. 94, 630 (1954).

[31] S. Meiboom and D. Gill, Modified spin-echo method for measuring nuclear relaxation times, Rev. Sci. Instrum. 29, 688 (1958).

[32] F. Mentink-Vigier, A. Collauto, A. Feintuch, I. Kaminker, V. Tarle, and D. Goldfarb, Increasing sensitivity of pulse EPR experiments using echo train detection schemes, J. Magn. Reson. 236, 117 (2013).

[33] T. Sekiguchi, A. M. Tyryshkin, S. Tojo, E. Abe, R. Mori, H. Riemann, N. V. Abrosimov, P. Becker, H.-J. Pohl, J. W. Ager, E. E. Haller, M. L. W. Thewalt, J. J. L. Morton, S. A. Lyon, and K. M. Itoh, Host isotope mass effects on the hyperfine interaction of group-V donors in silicon, Phys. Rev. B 90, 121203 (2014).

[34] V. Ranjan, S. Probst, B. Albanese, T. Schenkel, D. Vion, D. Esteve, J. Morton, and P. Bertet, Electron spin resonance spectroscopy with femtoliter detection volume, Appl. Phys. Lett. 116, 184002 (2020).

[35] E. Kupce and R. Freeman, Adiabatic pulses for wideband inversion and broadband decoupling, J. Magn. Reson. Ser. A (Print) 115, 273 (1995).
[36] A. J. Sigillito, H. Malissa, A. M. Tyryshkin, H. Riemann, N. V. Abrosimov, P. Becker, H. J. Pohl, M. L. Thewalt, K. M. Itoh, J. J. Morton, A. A. Houck, D. I. Schuster, and S. A. Lyon, Fast, low-power manipulation of spin ensembles in superconducting microresonators, Appl. Phys. Lett. 104, 222407 (2014).

[37] E. M. Purcell, in Confined Electrons and Photons (Springer, Boston, MA, 1995), p. 839.

[38] A. Bienfait, J. Pla, Y. Kubo, X. Zhou, M. Stern, C. Lo, C. Weis, T. Schenkel, D. Vion, D. Esteve, J. J. L. Morton, and P. Bertet, Controlling spin relaxation with a cavity, Nature 531, 74 (2016).

[39] G. A. Rinard, R. W. Quine, R. Song, G. R. Eaton, and S. S. Eaton, Absolute EPR spin echo and noise intensities, J. Magn. Reson. 140, 69 (1999).

[40] V. Ranjan, S. Probst, B. Albanese, A. Doll, O. Jacquot, E. Flurin, R. Heeres, D. Vion, D. Esteve, and J. Morton et al., Pulsed electron spin resonance spectroscopy in the Purcell regime, J. Magn. Reson. 310, 106662 (2020).

[41] K. Saeedi, S. Simmons, J. Z. Salvail, P. Dluhy, H. Riemann, N. V. Abrosimov, P. Becker, H.-J. Pohl, J. J. Morton, and M. L. Thewalt, Room-temperature quantum bit storage exceeding 39 min using ionized donors in silicon-28, Science 342, 830 (2013).

[42] L. Dreher, F. Hoehne, M. Stutzmann, and M. S. Brandt, Nuclear Spins of Ionized Phosphorus Donors in Silicon, Phys. Rev. Lett. 108, 027602 (2012).

[43] N. Iosad, B. Jackson, T. Klapwijk, S. Polyakov, P. Dmitirev, and J. Gao, Optimization of rf- and dcsputtered NbTiN films for integration with Nb-based SIS junctions, IEEE Trans. Appl. Supercond. 9, 1716 (1999).

[44] D. Glowacka, D. Goldie, S. Withington, H. Muhammad, G. Yassin, and B. Tan, Development of a $\mathrm{NbN}$ deposition process for superconducting quantum sensors, arXiv:1401.2292 (2014).

[45] J. Kroll, F. Borsoi, K. van der Enden, W. Uilhoorn, D. de Jong, M. Quintero-Pérez, D. van Woerkom, A. Bruno, S. Plissard, D. Car, E. Bakkers, M. Cassidy, and L. Kouwenhoven, Magnetic-Field-Resilient Superconducting Coplanar-Waveguide Resonators for Hybrid Circuit Quantum Electrodynamics Experiments, Phys. Rev. Appl. 11, 064053 (2019).

[46] C. Grèzes, Towards a Spin-Ensemble Quantum Memory for Superconducting Qubits: Design and Implementation of the Write, Read and Reset Steps (Springer International Publishing, Cham, Switzerland, 2015).

[47] B. Julsgaard and K. Mølmer, Reflectivity and transmissivity of a cavity coupled to two-level systems: Coherence properties and the influence of phase decay, Phys. Rev. A 85, 013844 (2012). 